Supplement of Atmos. Chem. Phys., 21, 4575-4597, 2021

https://doi.org/10.5194/acp-21-4575-2021-supplement

(c) Author(s) 2021. CC BY 4.0 License.

(C) (i)
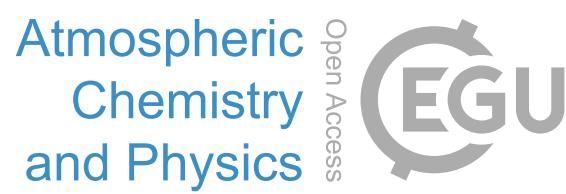

Supplement of

\title{
Turbulence-permitting air pollution simulation for the Stuttgart metropolitan area
}

Thomas Schwitalla et al.

Correspondence to: Thomas Schwitalla (thomas.schwitalla@uni-hohenheim.de)

The copyright of individual parts of the supplement might differ from the article licence. 


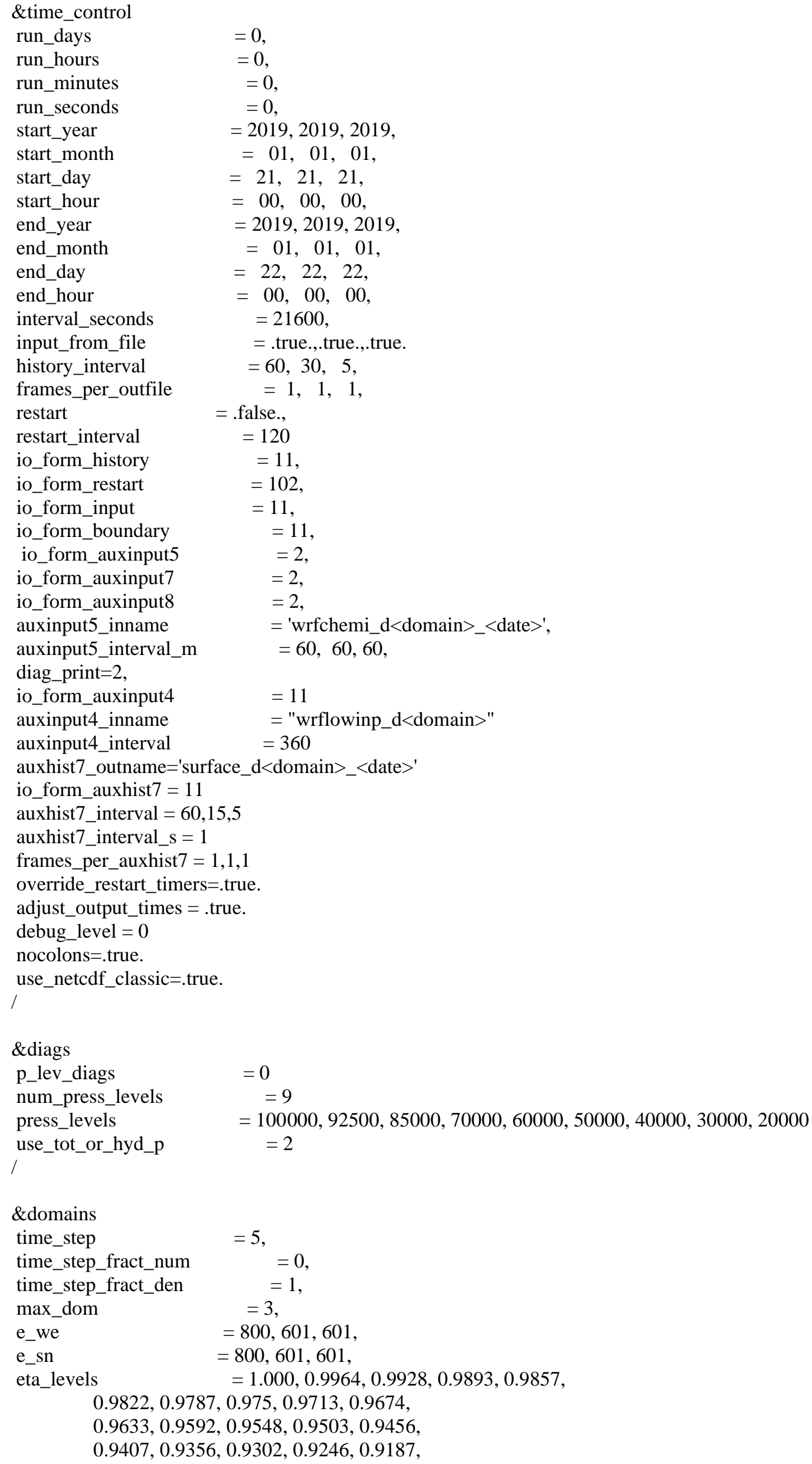

\&domains

time_step $\quad=5$

time_step_fract_num $\quad=0$, 


$$
\begin{aligned}
& \text { 0.9125, 0.906, 0.8992, 0.892, 0.8844, } \\
& 0.8764,0.868,0.8592,0.8499,0.84 \text {, } \\
& 0.8297,0.8188,0.8073,0.7952,0.7826 \text {, } \\
& \text { 0.7693, 0.7554, 0.7408, 0.7256, 0.7097, } \\
& 0.6932,0.6761,0.6583,0.6399,0.621 \text {, } \\
& 0.6016,0.5816,0.5612,0.5404,0.5193 \text {, } \\
& 0.498,0.4764,0.4548,0.4331,0.4114 \text {, } \\
& 0.3899,0.3685,0.3475,0.3268,0.3065 \text {, } \\
& 0.2867,0.2674,0.2488,0.2307,0.2111 \text {, } \\
& 0.1945,0.1789,0.1642,0.1505,0.1376 \text {, } \\
& 0.1256,0.1144,0.1041,0.0945,0.0856 \text {, } \\
& 0.0774,0.0699,0.0629,0.0565,0.0507 \text {, } \\
& 0.0453,0.0404,0.0359,0.0319,0.0281 \text {, } \\
& 0.0248,0.0217,0.0189,0.0164,0.0141 \text {, } \\
& 0.012,0.0101,0.0083,0.0068,0.0054 \text {, } \\
& \text { 0.0041, 0.0029, 0.0018, 0.0009, 0.000, } \\
& \text { e_vert } \\
& =100,100,100 \\
& \text { p_top_requested } \quad=5000 \text {. } \\
& \text { num_metgrid_levels } \quad=138 \text {, } \\
& \text { num_metgrid_soil_levels }=4 \text {, } \\
& \mathrm{dx} \quad=1250,250,50 \text {, } \\
& \text { dy } \quad=1250,250,50 \text {, } \\
& \text { grid_id } \quad=1,2,3 \text {, } \\
& \text { parent_id } \quad=0,1,2 \text {, } \\
& \text { i_parent_start } \quad=1,330,285 \text {, } \\
& \text { j_parent_start } \quad=1,355,208 \text {, } \\
& \text { parent_grid_ratio } \quad=1,5,5 \text {, } \\
& \text { parent_time_step_ratio } \quad=1,5,5 \text {, } \\
& \text { feedback }=0 \text {, } \\
& \text { smooth_option } \quad=0 \\
& \text { use_adaptive_time_step =.true. } \\
& \text { step_to_output_time =.true. } \\
& \text { max_step_increase_pct }=5,51,51 \\
& \text { starting_time_step }=500,100,50 \\
& \text { starting_time_step_den }=100,100,100 \\
& \text { max_time_step } \quad=1500,300,200 \\
& \text { max_time_step_den } \quad=100,100,100 \\
& \text { min_time_step } \quad=400,50,40 \\
& \text { min_time_step_den } \quad=100,100,100 \\
& \text { / } \\
& \text { \&physics } \\
& \text { sst_update } \quad=0 \text {, } \\
& \text { mp_physics } \quad=8,8,8 \text {, } \\
& \begin{array}{l}
\text { use_aero_icbc }=\text {.true. } \\
\text { ra_lw_physics }
\end{array}=4,4,4 \text {, } \\
& \text { ra_sw_physics }=4,4,4 \text {, } \\
& \text { radt } \quad=1,1,1 \text {, } \\
& \text { o3input }=2 \text {, } \\
& \text { sf_sfclay_physics } \\
& \text { sf_surface_physics } \\
& \text { bl_pbl_physics } \\
& \text { bldt } \\
& \text { topo_wind } \\
& \text { cu_physics } \\
& \text { cudt } \\
& \text { isfflx } \\
& \text { ifsnow } \\
& \text { icloud } \\
& \text { surface_input_source } \\
& \text { num_soil_layers } \\
& \text { mp_zero_out } \\
& \text { sf_urban_physics } \\
& =1,1,1 \text {, } \\
& =4,4,4 \text {, } \\
& =1,0,0 \text {, } \\
& =0,0,0 \text {, } \\
& =2,2,2 \\
& =0,0,0 \text {, } \\
& =5,0,0 \text {, } \\
& =1 \text {, } \\
& =1 \text {, } \\
& =1 \text {, } \\
& =3 \text {, } \\
& =4 \text {, } \\
& =0 \text {, } \\
& =1,1,1
\end{aligned}
$$




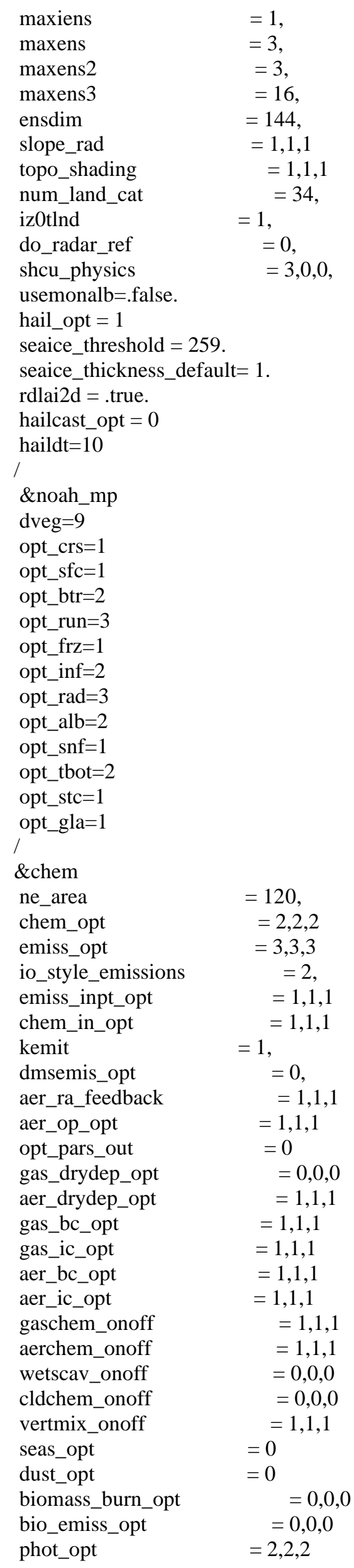




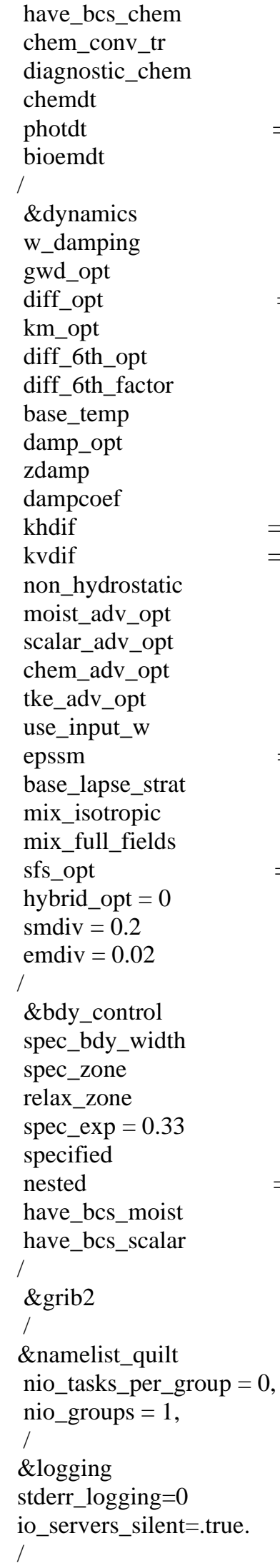

